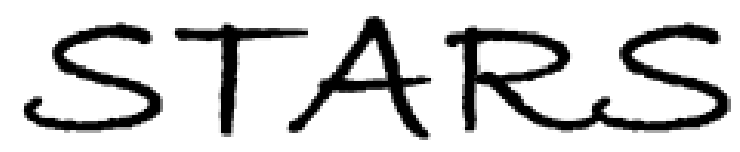

University of Central Florida

STARS

$1-1-2008$

\title{
Translocation dynamics with attractive nanopore-polymer interactions
}

\author{
Kaifu Luo \\ Tapio Ala-Nissila \\ See-Chen Ying \\ Aniket Bhattacharya \\ University of Central Florida
}

Find similar works at: https://stars.library.ucf.edu/facultybib2000 University of Central Florida Libraries http://library.ucf.edu

This Article is brought to you for free and open access by the Faculty Bibliography at STARS. It has been accepted for inclusion in Faculty Bibliography 2000s by an authorized administrator of STARS. For more information, please contactSTARS@ucf.edu.

\section{Recommended Citation}

Luo, Kaifu; Ala-Nissila, Tapio; Ying, See-Chen; and Bhattacharya, Aniket, "Translocation dynamics with attractive nanopore-polymer interactions" (2008). Faculty Bibliography 2000 s. 660.

https://stars.library.ucf.edu/facultybib2000/660

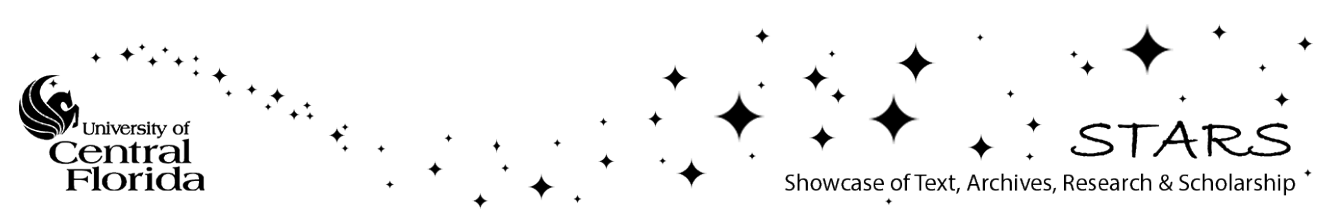




\title{
Translocation dynamics with attractive nanopore-polymer interactions
}

\author{
Kaifu Luo, ${ }^{1,2, *}$ Tapio Ala-Nissila, ${ }^{2,3}$ See-Chen Ying, ${ }^{3}$ and Aniket Bhattacharya ${ }^{4}$ \\ ${ }^{1}$ Physics Department, Technical University of Munich, D-85748 Garching, Germany \\ ${ }^{2}$ Department of Applied Physics, Helsinki University of Technology, P. O. Box 1100, FIN-02015 TKK, Espoo, Finland \\ ${ }^{3}$ Department of Physics, Box 1843, Brown University, Providence, Rhode Island 02912-1843, USA \\ ${ }^{4}$ Department of Physics, University of Central Florida, Orlando, Florida 32816-2385, USA \\ (Received 13 May 2008; revised manuscript received 15 August 2008; published 19 December 2008)
}

\begin{abstract}
Using Langevin dynamics simulations, we investigate the influence of polymer-pore interactions on the dynamics of biopolymer translocation through nanopores. We find that an attractive interaction can significantly change the translocation dynamics. This can be understood by examining the three components of the total translocation time $\tau \approx \tau_{1}+\tau_{2}+\tau_{3}$ corresponding to the initial filling of the pore, transfer of polymer from the cis side to the trans side, and emptying of the pore, respectively. We find that the dynamics for the last process of emptying of the pore changes from nonactivated to activated in nature as the strength of the attractive interaction increases, and $\tau_{3}$ becomes the dominant contribution to the total translocation time for strong attraction. This leads to nonuniversal dependence of $\tau$ as a function of driving force and chain length. Our results are in good agreement with recent experimental findings, and provide a plausible explanation for the different scaling behavior observed in solid state nanopores vs that for the natural $\alpha$-hemolysin channel.
\end{abstract}

DOI: 10.1103/PhysRevE.78.061918

PACS number(s): 87.15.A-, 87.15.H-

\section{INTRODUCTION}

The controlled transport of polymer molecules through a nanopore has received increasing attention due to its importance in biological systems and its potentially revolutionary technological applications [1,2]. There is a flurry of experimental [3-24] and theoretical [24-70] studies devoted to this subject. In an important experiment, Kasianowicz et al. [1] demonstrated that an electric field can drive single-stranded DNA and RNA molecules through the water-filled $\alpha$-hemolysin channel and that the passage of each molecule is signaled by a blockade in the channel current. These observations can be used to directly characterize the polymer length. Similar experiments have been done recently using solid state nanopores with more precisely controlled dimensions [15-24]. Currently, extensive effort is being made to unravel the dependence of the translocation time $\tau$ on the system parameters such as the polymer chain length $N \quad[5,6,24,26,29,30,32,35,41-53,55-57,64]$, pore length $L$ and pore width $W$ [48], driving force $F$ $[5,6,9,11,35,38,39,42,49,50,55-57,64]$, sequence and secondary structure $[3,4,6,52,53,55]$, and polymer-pore interactions $[4,6,32,53-55,64,67]$.

Recent experiments $[3,4,6,12-14]$ have shown that different DNA polymers can be distinguished from each other. In particular, Meller et al. $[4,6]$ have shown how several different DNA polymers can be identified by a unique pattern in an "event diagram." The event diagrams are plots of translocation duration versus blockade current for an ensemble of events. Patterns for a given polymer can be characterized uniquely by the blockade current, the translocation time, and its distribution. Because each type of polynucleotide gives rise to specific values of these three parameters, DNA molecules which differ from each other only by sequence can be

\footnotetext{
*Corresponding author; luokaifu@gmail.com
}

distinguished. At room temperature striking differences were found for the translocation time distributions of polydeoxyadenylic acid $\left[\left(\operatorname{poly}(\mathrm{dA})_{100}\right)\right]$ and polydeoxycytidylic acid $\left[\left(\operatorname{poly}(\mathrm{dA})_{100}\right)\right]$ DNA molecules. The translocation time of poly $(\mathrm{dA})$ is found to be much longer, which agrees with other experiments $[3,12]$, and its distribution is wider with a longer tail compared with the corresponding data for poly (dC). Moreover, the differences between the translocation behavior are accentuated at lower temperature. The origin of the different behavior was attributed to stronger attractive interaction of poly $(\mathrm{dA})$ with the pore.

Recently, Robertson et al. [71] and Krasilnikov et al. [72] have investigated the dynamics of single neutral poly (ethylene glycol) (PEG) molecules in the $\alpha$-hemolysin channel. Robertson et al. [71] showed that the different size polymers in polydisperse sample can be distinguished based on quantitative information on residence times in the $\alpha$-hemolysin channel. In the limit of a strong attractive polymer-pore attraction, Krasilnikov et al. [72] observed that the residence time in the channel shows a novel nonmonotonic behavior as a function of the molecular weight.

The other experimental data that point to the possible essential role of the monomer-pore interaction concerns the different conflicting values of scaling exponents of $\tau$ with $N$ and with the applied voltage as reported in recent experiments. A linear dependence $\tau \sim N$ was observed for polymer translocation through the $\alpha$-hemolysin channel [1,5], while another experiment reported that $\tau \sim N^{1.27} \approx N^{2 \nu}$ for a synthetic nanopore [24], where $\nu$ is the Flory exponent $[73,74]$. As to the dependence of the translocation time on the applied voltage for the $\alpha$-hemolysin channel, an inverse linear behavior [1] is observed for polyuridylic acid [poly (U)] while an inverse quadratic dependence [5] is found for polydeoxyadenylic acid. One possible explanation for all these conflicting data is that the polymer-pore interaction depends crucially on the details of the pore structure ( $\alpha$-hemolysin channel vs synthetic nanopore) in addition to being base pair specific. 
To date, most of the theoretical studies of the translocation of biopolymers through nanopre are based on models in which the wall of the pore plays only a passive role in confining the polymer to the inside of the pore. There are only a few theoretical studies of such interaction effects. Based on a Smoluchowski equation with a phenomenological microscopic potential to describe the polymer-pore interactions, Lubensky and Nelson [32] captured the main ingredients of the translocation process. However, when compared with experiments, their model is not sufficient. Numerically, Tian and Smith [64] found that attraction facilitates the translocation process by shortening the translocation time, which contradicts experimental findings [4,6]. In a recent Letter [53], we used Langevin dynamics simulations to investigate the influence of polymer-pore interactions on translocation. We found that with increasing attraction the histogram for the translocation time $\tau$ shows a transition from a Gaussian to a long-tailed distribution corresponding to thermal activation over a free energy barrier. The $N$ dependence of the entropic force leads to both the translocation time and the residence time in the pore showing a nonmonotonic behavior as a function of $N$ for short chains in the strong-attraction limit. These results are in good agreement with the above experimental data $[4,6,12,72]$.

In the present work, we further show that strong polymerpore interactions can directly affect the effective scaling exponents of $\tau$ both with $N$ and with the applied voltage, which provides a possible explanation for the different experimental findings $[1,5,24]$ on these physical quantities. We provide a microscopic understanding of how strong polymer-pore interaction influences the translocation dynamics. This is done through analyzing the three quantities $\tau_{1}, \tau_{2}$, and $\tau_{3}$ corresponding to initial filling of the pore, transfer of the polymer from the cis side to the trans side, and finally emptying of the pore, respectively. We find that the final process of emptying the pore, $\tau_{3}$, involves an activation barrier and completely dominates the translocation time in the strongattractive-interaction limit. This leads to a strong dependence of the effective scaling exponents associated with the translocation time on both the strength of the attraction and the driving force. In addition, we examine the waiting time and residence time distributions. These quantities are related to the translocation time but the waiting time provides more detailed information about the translocation dynamics, while the residence time is the more relevant quantity for direct comparison with the experimental observations.

\section{MODEL AND METHODS}

In our numerical simulations, the polymer chains are modeled as bead-spring chains of Lennard-Jones (LJ) particles with the finite extension nonlinear elastic (FENE) potential. Excluded volume interaction between monomers is modeled by a short-range repulsive LJ potential: $U_{\mathrm{LJ}}(r)$ $=4 \varepsilon\left[(\sigma / r)^{12}-(\sigma / r)^{6}\right]+\varepsilon$ for $r \leqslant 2^{1 / 6} \sigma$ and 0 for $r>2^{1 / 6} \sigma$. Here, $\sigma$ is the diameter of a monomer, and $\varepsilon$ is the depth of the potential. The connectivity between neighboring monomers is modeled as a FENE spring with $U_{\mathrm{FENE}}(r)=$ $-\frac{1}{2} k R_{0}^{2} \ln \left(1-r^{2} / R_{0}^{2}\right)$, where $r$ is the distance between con-

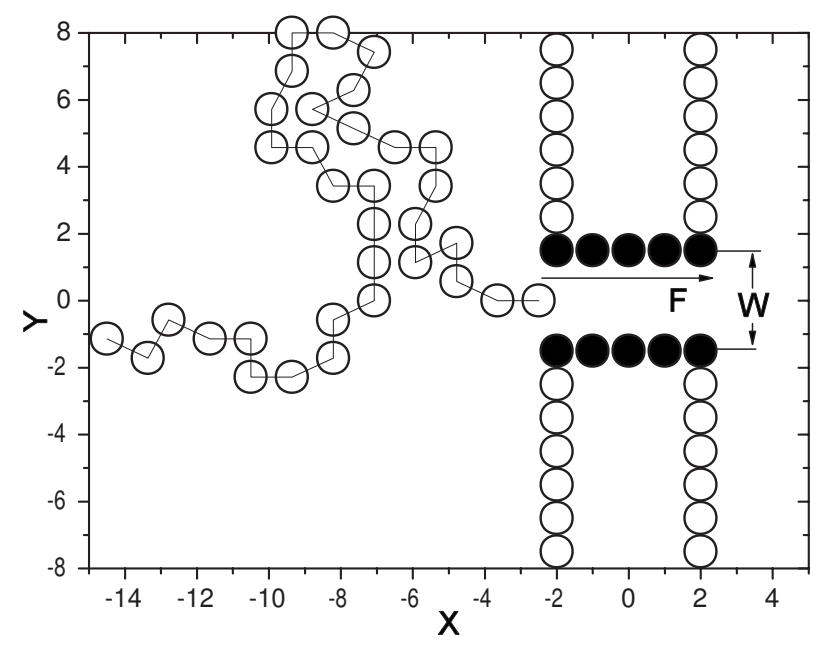

FIG. 1. Schematic representation of the system. The pore length $L=5$ and the pore width $W=3$ (see text for the units).

secutive monomers, $k$ is the spring constant, and $R_{0}$ is the maximum allowed separation between connected monomers.

We consider a two-dimensional (2D) geometry as shown in Fig. 1, where the wall in the $y$ direction is formed by stationary particles within a distance $\sigma$ from each other. The pore of length $L$ and width $W$ in the center of the wall is composed of stationary black particles. Between all monomer-wall particle pairs, there exists the same shortrange repulsive $\mathrm{LJ}$ interaction as described above. The poremonomer interaction is modeled by a LJ potential with a cutoff of $2.5 \sigma$ and interaction strength $\varepsilon_{\mathrm{pm}}$. This interaction can be either attractive or repulsive depending on the position of the monomer with respect to the pore particles. In the Langevin dynamics simulation, each monomer is subjected to conservative, frictional, and random forces, respectively, with [75] $m \ddot{\mathbf{r}}_{i}=-\boldsymbol{\nabla}\left(U_{\mathrm{LJ}}+U_{\mathrm{FENE}}\right)+\mathbf{F}_{\mathrm{ext}}-\xi \mathbf{v}_{i}+\mathbf{F}_{i}^{R}$, where $m$ is the monomer's mass, $\xi$ is the friction coefficient, $\mathbf{v}_{i}$ is the monomer's velocity, and $\mathbf{F}_{i}^{R}$ is the random force which satisfies the fluctuation-dissipation theorem. The external force is expressed as $\mathbf{F}_{\mathrm{ext}}=F \hat{\mathbf{x}}$, where $F$ is the external force strength exerted on the monomers in the pore, and $\hat{\mathbf{x}}$ is a unit vector in the direction along the pore axis.

In the present work, we use the LJ parameters $\varepsilon$ and $\sigma$ and the monomer mass $m$ to fix the energy, length, and mass scales, respectively. The time scale is then given by $t_{\mathrm{LJ}}$ $=\left(m \sigma^{2} / \varepsilon\right)^{1 / 2}$. The dimensionless parameters in our simulations are $R_{0}=2, k=7, \xi=0.7$, and $k_{B} T=1.2$ unless otherwise stated. For the pore, we set $L=5$ unless otherwise stated. The width $W$ is set to the value 3 . This ensures that the polymer encounters an attractive force inside the pore [76]. The driving force $F$ is set between 0.5 and 2.0, which correspond to the range of voltages used in the experiments $[1,5]$. The Langevin equation is integrated in time by a method described by Ermak and Buckholz [77] in 2D. Initially, the first monomer of the chain is placed in the entrance of the pore, while the remaining monomers are under thermal collisions described by the Langevin thermostat to obtain an equilibrium configuration. Typically, we average our data over 2000 independent runs. 


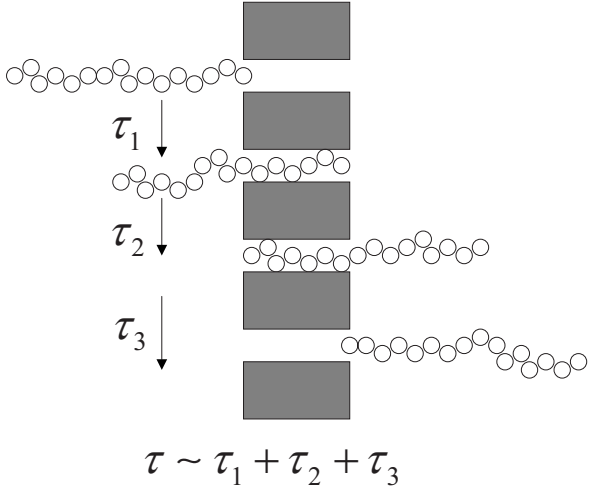

FIG. 2. Three components of the translocation process.

\section{RESULTS AND DISCUSSION}

\section{A. Translocation time, waiting time, and residence time}

The translocation time is defined as the time interval between the entrance of the first segment into the pore and the exit of the last segment. We can break down the translocation process into three components, as shown in Fig. 2. The total translocation time $\tau$ can be written as a sum of three contributions $\tau \approx \tau_{1}+\tau_{2}+\tau_{3}$, where $\tau_{1}, \tau_{2}$, and $\tau_{3}$ correspond to initial filling of the pore, transfer of the polymer from the cis side to the trans side, and finally emptying of the pore, respectively. To shed light on the detailed translocation process, we examine the number of translocated monomers $n_{\text {trans }}$ as a function of the time for a typical successful translocation event for $N=128$, and two values of the monomer attractive interaction strength. The value $\varepsilon_{\mathrm{pm}}=1.0$ corresponds to a weak interaction whereas $\varepsilon_{\mathrm{pm}}=3.0$ corresponds to the strongattraction limit. Here, $n_{\text {trans }}=0$ before the first monomer exits the pore and $n_{\text {trans }}=N$ after the last monomer has threaded through the pore. As shown in Fig. 3, under the weak driving force $F=0.5, \tau_{1}$ is not sensitive to the attraction strength and $\tau_{1} \ll \tau_{2}$. $\tau_{2}$ for the strong attraction with $\varepsilon_{\mathrm{pm}}=3.0$ is roughly twice that for the weak attraction with $\varepsilon_{\mathrm{pm}}=1.0$. However, $\tau_{3}$

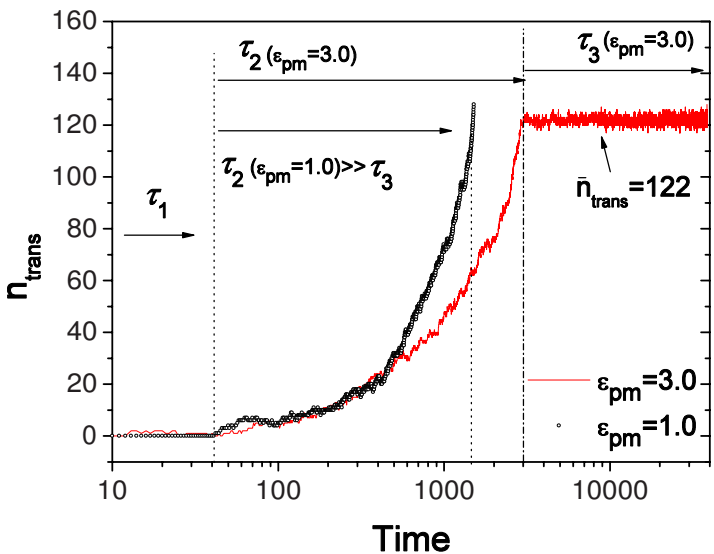

FIG. 3. (Color online) Number of translocated monomers $n_{\text {trans }}$ as a function of the time for $\varepsilon_{\mathrm{pm}}=1.0$ and 3.0 under the driving force $F=0.5$. For both strong and weak attraction strengths, $\tau_{1} \ll \tau$. For weak attraction strength $\varepsilon_{\mathrm{pm}}=1.0$, we find $\tau_{3} \ll \tau_{2}$ and thus $\tau$ $\approx \tau_{2}$.

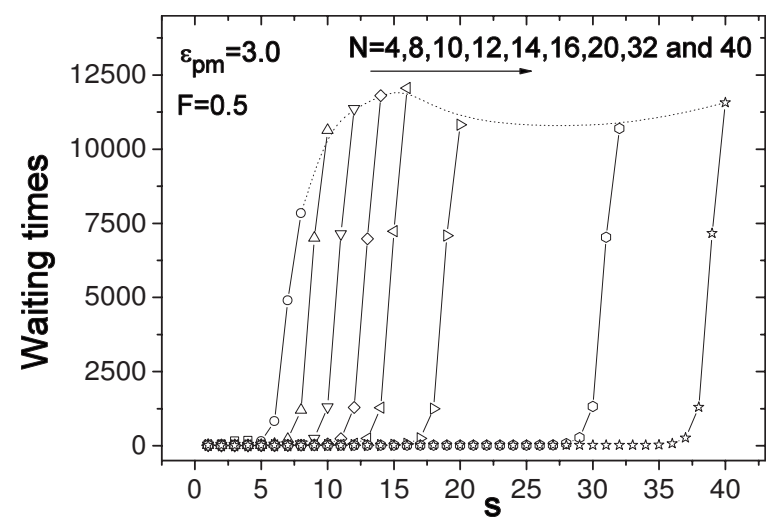

FIG. 4. Waiting time of different chain lengths for $\varepsilon_{\mathrm{pm}}=3.0$ and $F=0.5$.

depends strongly on the attraction strength. For $\varepsilon_{\mathrm{pm}}=1.0$, $\tau_{3} \ll \tau_{2}$ and is basically negligible for the pore length $L=5$. For the strong-attraction limit with $\varepsilon_{\mathrm{pm}}=3.0$, the situation is totally different, with $\tau_{3}$ more than an order of magnitude larger than $\tau_{2}$, completely dominating the total contribution to the translocation time. From Fig. 3, it can be seen that the number of translocated monomers oscillates around $n_{\text {trans }}$ $\approx 122$, which corresponds to the beginning of the last stage of the translocation process, namely, the emptying of the pore. This is due to the activated nature of the translocation process with a free energy difference of $\Delta \tilde{F}=L\left[\varepsilon_{\mathrm{pm}}-F / 2\right.$ $-f(N)]$ between the final and the initial state. The term $f(N)$ here accounts for the entropic driving force which should take effect at larger values of $N$, and eventually saturate for very long polymers. This leads to the long oscillation time of the last few monomers with repeated forward and backward motions. The final emptying of the pore corresponds to a rare crossing of the barrier.

To provide more microscopic details of the translocation process, we investigate the waiting time distribution for different chain lengths $N$ in the strong-attraction limit. The waiting time of monomer $s$ is defined as the average time between the events where monomer $s$ and monomer $s+1$ exit the pore. In our previous work $[49,50]$ for pure repulsive monomer-pore interactions, we found that the waiting time depends strongly on the monomer positions in the chain. For relatively short polymers, the monomers in the middle of the polymer need the longest time to exit the pore. Here, the waiting time of different chain lengths for $\varepsilon_{\mathrm{pm}}=3.0$ and $F$ $=0.5$ are shown in Fig. 4. It can be seen that it takes a much longer time for the last three monomers to exit the pore, which is completely different behavior from that for pure repulsive monomer-pore interactions. This behavior correlates with the oscillation of the last monomers as shown in Fig. 3. Here we should mention that due to the entropic factor $f(N)$ in the barrier the waiting time for these last few monomers actually decreases in the range $N \approx 14-32$ before saturating and even increasing slightly with further increase of $N$.

For a successful translocation, as noted above, the system must overcome a free energy difference of $\Delta \widetilde{F}=L\left[\varepsilon_{\mathrm{pm}}\right.$ $-F / 2-f(N)]$. As a result, there exists a strong voltage de- 

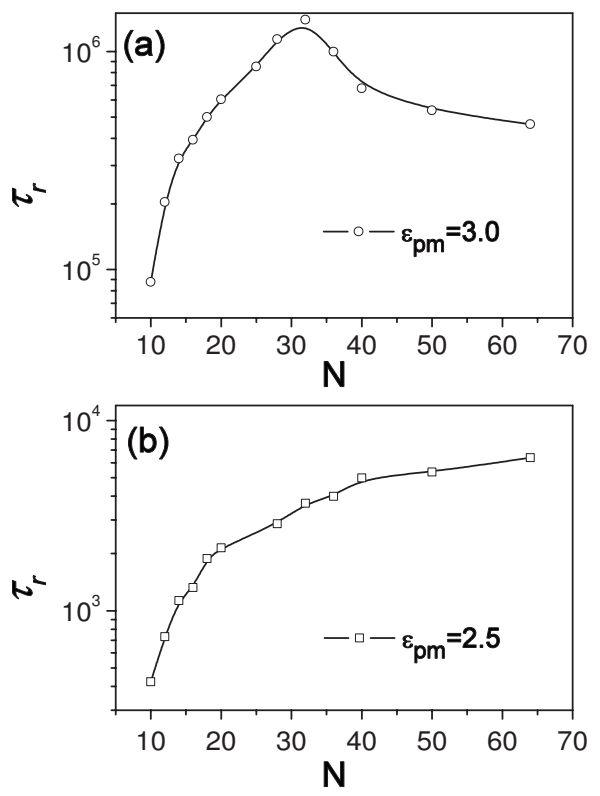

FIG. 5. Residence time $\tau_{r}$ as a function of the chain length for $\varepsilon_{\mathrm{pm}}=$ (a) 3.0 and (b) 2.5 under the driving force $F=0$.

pendence of a single-stranded DNA entering and transporting through the $\alpha$-hemolysin pore $[11,36]$. Under zero and low driving forces, the translocation probability is very small in the sense that, for many translocation events, once they are started they do not finish all the way. Instead, the polymer returns and exits to the cis side again. This means that the $\tau_{1}$ process of filling the pore does not get completed and the real translocation process corresponding to $\tau_{2}$ and $\tau_{3}$ never even gets started. We define an additional residence time $\tau_{r}$ as the weighted average of the translocation time for the completed events and the return time for the events that start and return via the cis side. The significance of $\tau_{r}$ is that it corresponds to the experimentally measured average blockage time of the polymer in the nanopore, which does not distinguish return events from the completed translocated events. For zero or low driving force $(F<0.5)$, the residence time is almost completely dominated by return events. We have calculated the residence time $\tau_{r}$ for $\varepsilon_{\mathrm{pm}}=3.0$ and 2.5 in Fig. 5. As shown in Ref. [53], in the strong-attraction case with $\varepsilon_{\mathrm{pm}}=3.0$, the $N$ dependence of the residence time here is nonmonotonic [see Fig. 5(a)]. This result for $\tau_{r}$ is in good agreement with experimental data of Krasilnikov et al. [72] where the residence time of a neutral PEG molecule in an $\alpha$-hemolysin pore was measured. Here, we further show that, for $\varepsilon_{\mathrm{pm}}=2.5, \tau_{r}$ increases with increasing $N$ [see Fig. 5(b)]. It indicates that the strong attraction plays an essential role in the observed nonmonotonic behavior.

For $\varepsilon_{\mathrm{pm}}=3.0$, the distribution of $\tau_{r}$ is shown in Fig. 6. One obvious feature is the existence of two groups. The first group with shorter $\tau_{r}$ corresponds to the events where one end of the chain accesses the pore, and then quickly returns back. For the second group with longer $\tau_{r}$, the residence time is still about $99.8 \%$ due to return events for $\varepsilon_{\mathrm{pm}}=3.0$. In the strong-attraction limit, once the attractive force reaches its maximum when the pore is fully filled by monomers, it takes a very long time for the polymer to return back due to frequent backward and forward events.

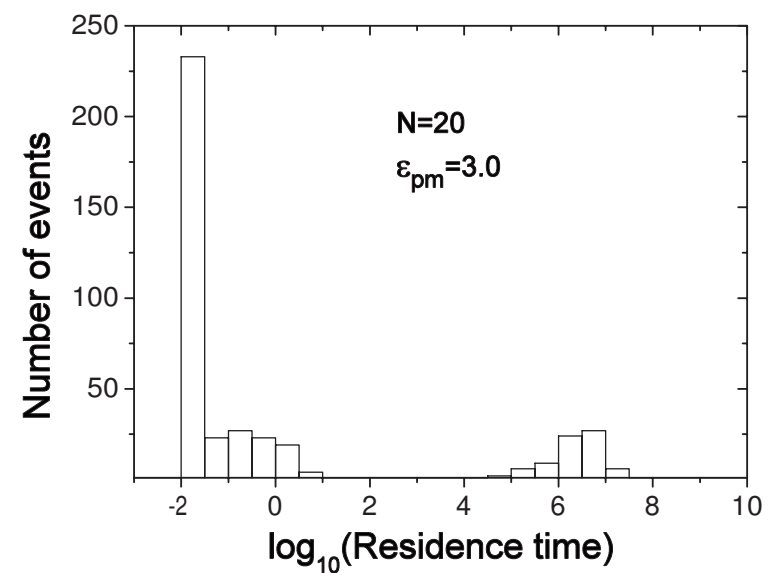

FIG. 6. Distribution of the residence time $\tau_{r}$ for $\varepsilon_{\mathrm{pm}}=3.0$ and $F=0$. The chain length $N=20$.

\section{B. Dependence of translocation time on various parameters}

\section{Translocation time as a function of temperature}

Figure 7 shows the translocation time $\tau$ as a function of the temperature for different attraction strengths. For the whole examined range of temperatures, $\tau$ decreases very slightly with increasing temperature for a weak attractive strength of $\varepsilon_{\mathrm{pm}}=1.0$. However, for the strong attractive strength $\varepsilon_{\mathrm{pm}}=3.0$, with increasing temperature $\tau$ first rapidly decreases and then approaches saturation at higher temperatures. At higher temperatures, the differences between translocation times for weak and strong attractive strengths become very small. This temperature dependence of translocation time is in good agreement with experiments [4].

\section{Translocation time as a function of the driving force}

In the weak-attraction (i.e., nonactivated) region, the overall $\tau$ is determined mainly by $\tau_{2}$ and its dependence on the driving force scales as $F^{-1}$. This simple scaling behavior can be understood by considering the steady state of motion of the polymer through the nanopore. The average velocity is determined by balancing the frictional damping force (pro-

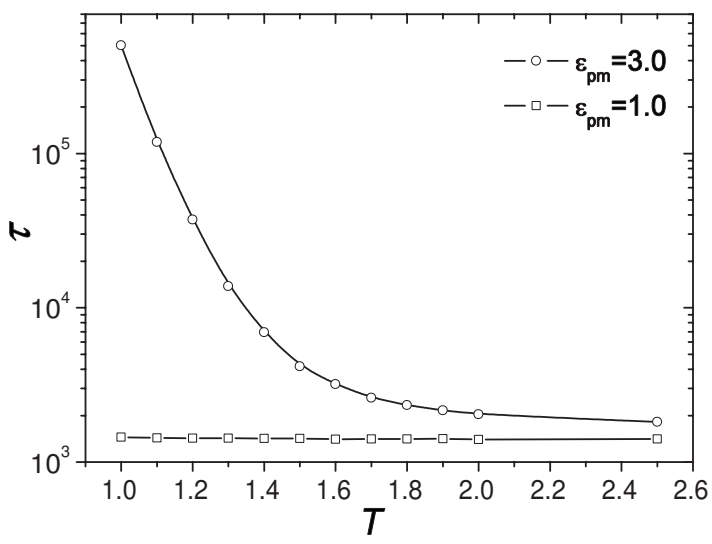

FIG. 7. Translocation time as a function of the temperature for both strong and weak attraction $\left(\varepsilon_{\mathrm{pm}}=3.0\right.$ and 1.0 , respectively) under the driving force $F=0.5$. The chain length $N=128$. 


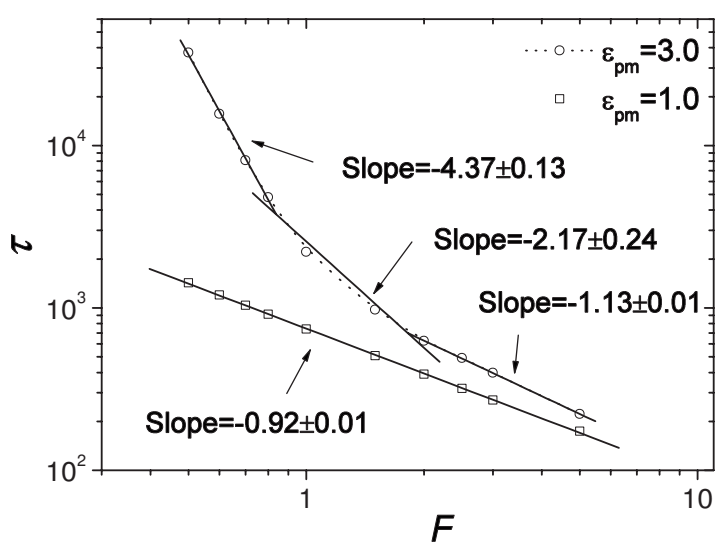

FIG. 8. Translocation time as a function of the driving forces for both strong and weak attraction, $\varepsilon_{\mathrm{pm}}=3.0$ and 1.0. The chain length $N=128$.

portional to the velocity) with the external driving force. This leads to an average velocity proportional to the driving force $F$, and hence a translocation time $\tau \sim F^{-1}$. In Fig. 8 we show the dependence of the translocation time $\tau$ on the driving force. It can be seen that in the weak-interaction limit for $\varepsilon_{\mathrm{pm}}=1.0$ the data are very close to the linear scaling behavior $\tau \sim F^{-1}$ as predicted. For a strong attractive interaction with $\varepsilon_{\mathrm{pm}}=4.0$, the situation is more complicated. For weak driving forces $(F \leqslant 2)$, one is in the activated region where the inverse of the translocation time obeys an Arrhenius form. However, the driving force $F$ affects both the activation barrier and the prefactor, leading to a complicated dependence of $\tau$ on the driving force that does not have a simple power law scaling form as seen in Fig. 8 for the $\varepsilon_{\mathrm{pm}}=3.0$ result. Insistence on fitting the data with a power law scaling form will lead to an effective scaling exponent that changes with the value of the driving force. Finally, beyond a critical force, the activation barrier disappears and one should obtain asymptotically the $\tau \sim F^{-1}$ behavior just as in the weakinteraction case. This whole scenario is very similar to the sliding friction of an adsorbed layer under an external driving force [78].

The above theoretical considerations lead to a possible explanation of recent apparently conflicting experimental data. Polyuridylic acid has a weak interaction with the pore, and it is not surprising that an inverse linear dependence of the translocation time on applied voltage was observed in experiments on the translocation of poly(U) [1]. However, poly $(\mathrm{dA})$ has a much stronger interaction with the pore compared with poly(U). Thus it should be in the stronginteraction activated region with a larger effective scaling exponent. Indeed, an inverse quadratic dependence of the translocation time on applied voltage was experimentally observed for poly (dA) [5]. It would be desirable to have measurement made over a larger range of the applied voltage to critically test our predictions for the effective scaling exponent.

\section{Translocation time as a function of chain length}

Previously, we have established that, for pure repulsive polymer-pore interactions, the dependence of the transloca-

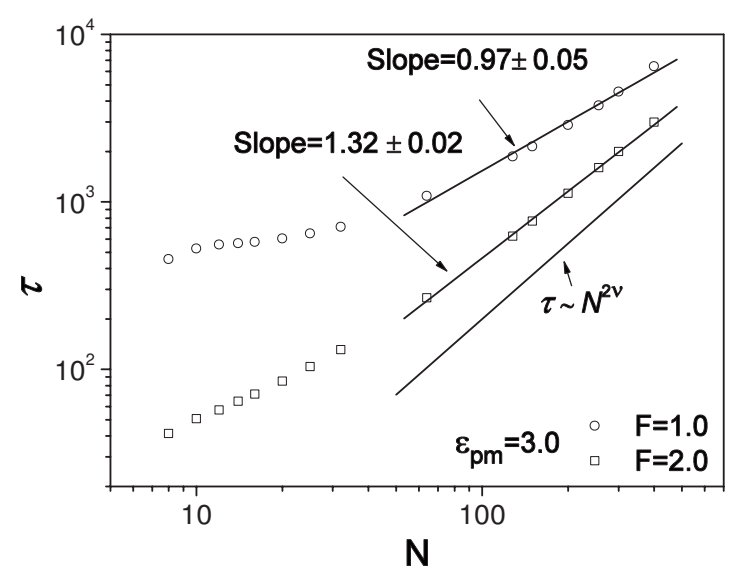

FIG. 9. Translocation time as a function of the chain length for $\varepsilon_{\mathrm{pm}}=3.0$ under $F=1.0$ and 2.0 , respectively.

tion time on the length of the polymer scales as $\tau \sim N^{2 \nu}$ for $N<200$ and crosses over to a new scaling regime $\tau \sim N^{1+\nu}$ for larger values of $N[49,50,52]$. In the presence of weak interaction between the monomer and the pore, the qualitative dependence on the length of the polymer remains the same. For stronger attractive strength $\varepsilon_{\mathrm{pm}}=3.0$, the scaling exponent of $\tau$ with $N$ for $64 \leqslant N \leqslant 400$ becomes strongly dependent on the driving force, with no indication of crossover behavior, as shown in Fig. 9. We find $\tau \sim N^{1.32}$ for $F$ $=2.0$, which is close to $\tau \sim N^{2 \nu}$ with the Flory exponent $\nu$ $=0.75$ in $2 \mathrm{D}[73,74]$, and $\tau \sim N^{0.97}$ for $F=1.0$. The dependence on the length of the polymer is due to the change from the nonactivated regime for weak attractive or pure repulsive interaction to an activated regime for strong attractive interaction.

Experimentally, a linear dependence $\tau \sim N$ was observed in experiments $[1,5]$ for polymer translocation through the $\alpha$-hemolysin channel, in contrast to the $\tau \sim N^{2 \nu}$ scaling observed for polymer translocation through the solid-state nanopore [24]. This difference can be understood in light of our present results concerning the influence of the different polymer-pore interaction on the length dependence of the translocation time. For a synthetic pore, there is at most a very weak attractive interaction between the polymer and the pore, and one expects the scaling behavior $\tau \sim N^{2 \nu}$ to hold for $N \leqslant 200$. However, a stronger attractive interaction is expected to exist between the different bases and the $\alpha$-hemolysin channel. For the models studied in this work, it changes the scaling behavior from $\tau \sim N^{2 \nu}$ to $\tau \sim N$. This provides a possible explanation for the difference of the experimental observations in the different nanopores $[1,5,24]$.

Under a strong attractive force with $\varepsilon_{\mathrm{pm}}=3.0$ and a weak driving force $F=0.5$, the translocation time $\tau$ has a qualitatively different dependence on $N$ as compared with the pure repulsive or weak attractive pore interaction. Here we should mention that for $F=0.5$ we cannot access $N>128$, as the translocation time becomes too long to be feasible for numerical computation. As shown earlier in Ref. [53] and here in Fig. 10(a), the translocation time displays nonmonotonic behavior with a rapid increase to a maximum at $N \sim 14$, followed by a decrease for $14<N<32$, and an increase again for $N>32$. The eventual increase in the large $-N$ limit is due 

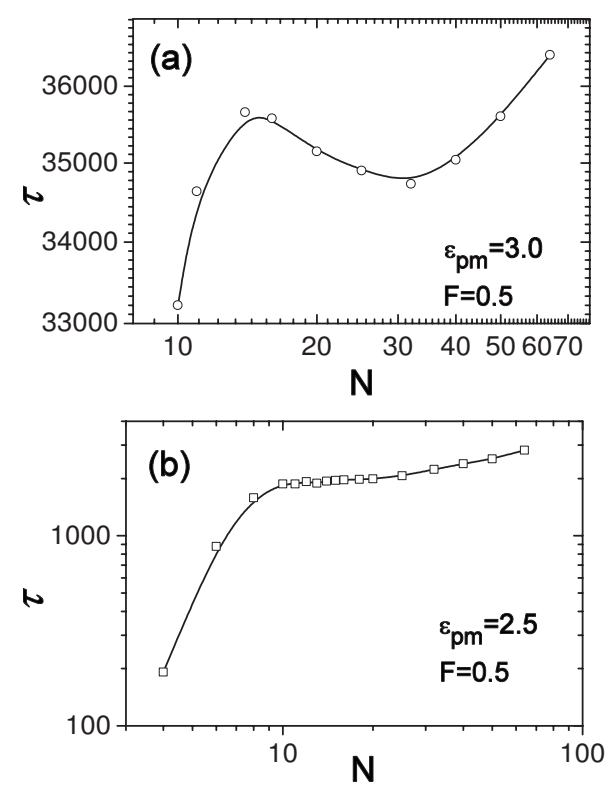

FIG. 10. Translocation time $\tau$ as a function of the chain length for $\varepsilon_{\mathrm{pm}}=$ (a) 3.0 and (b) 2.5 under the driving force $F=0.5$.

to the $\tau_{2}$ contribution for longer chains. The observed nonmonotonic behavior is also reflected qualitatively in the waiting time distribution as shown in Fig. 4. As shown in Fig. $10(\mathrm{~b})$, when the attractive force is decreased to $\varepsilon_{\mathrm{pm}}=2.5$, this nonmonotonic behavior vanishes.

To understand the microscopic origin of the translocation dynamics shown in Fig. 10, in Fig. 11 we show $\tau_{1}+\tau_{2}$ as a function of the chain length for different attraction strengths under the driving force $F=0.5$. For $32 \leqslant N \leqslant 200, \tau_{1}+\tau_{2}$ $\sim N^{2 \nu}$ is observed, irrespective of attraction strengths. This indicates that the nonmonotonic behavior shown in Fig. 10 in the strong-interaction limit is again due to the pore-emptying process corresponding to $\tau_{3}$ dominating the translocation time in the strong-interaction limit.

\section{CONCLUSIONS}

In this work, we have studied the dependence of the translocation time on the temperature, attraction strength, driving force, and chain length. To analyze the influence of the attractive interaction in more detail, we have considered the three components of the translocation time $\tau \approx \tau_{1}+\tau_{2}+\tau_{3}$,

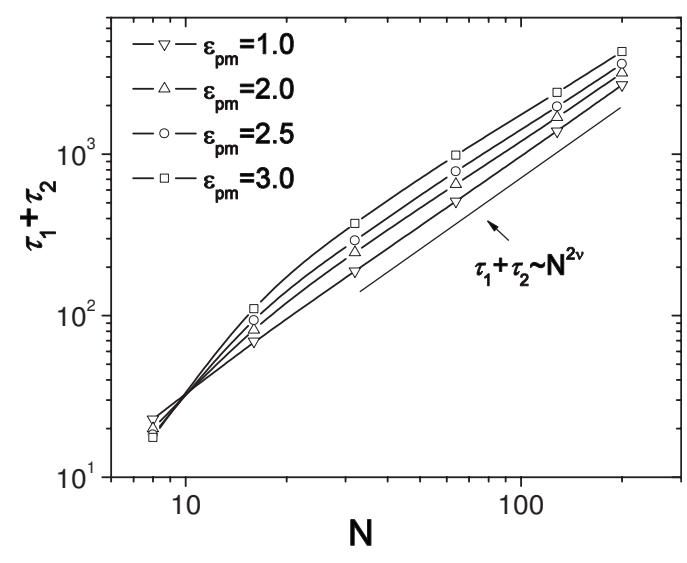

FIG. 11. $\tau_{1}+\tau_{2}$ as a function of the chain length for different $\varepsilon_{\mathrm{pm}}$ under the driving force $F=0.5$.

which were examined as a function of the attraction strength. Here $\tau_{1}, \tau_{2}$, and $\tau_{3}$ correspond to initial filling of the pore, transfer of polymer from the cis side to the trans side, and emptying of the pore, respectively. We find that $\tau_{1} \ll \tau_{2}$ for both weak and strong attraction strengths, for $N$ in the typical range used in the experiments. However, $\tau_{3}$ is sensitive to the presence of an attractive interaction and changes from a value much less than $\tau_{2}$ for weak interactions to the dominant contribution to the overall translocation time due to the rare activated event nature of the final emptying of the pore. This leads to a drastic change of the translocation dynamics and various scaling exponents as a function of the strength of the attractive monomer pore interactions. Our theoretical results are in good agreement with recent experimental data $[4,6,72]$. They also provide a possible explanation for the difference of the scaling behaviors with regard to the driving force and the length of polymers observed using different types of nanopores $[1,5,24]$.

\section{ACKNOWLEDGMENTS}

This work has been supported in part by The Academy of Finland through its Center of Excellence (COMP) and TransPoly Consortium grants. We wish to thank CSC Ltd. for allocation of computer resources.
[1] J. J. Kasianowicz, E. Brandin, D. Branton, and D. W. Deamer, Proc. Natl. Acad. Sci. U.S.A. 93, 13770 (1996).

[2] A. Meller, J. Phys.: Condens. Matter 15, R581 (2003).

[3] M. Akeson, D. Branton, J. J. Kasianowicz, E. Brandin, and D. W. Deamer, Biophys. J. 77, 3227 (1999).

[4] A. Meller, L. Nivon, E. Brandin, J. Golovchenko, and D. Branton, Proc. Natl. Acad. Sci. U.S.A. 97, 1079 (2000).

[5] A. Meller, L. Nivon, and D. Branton, Phys. Rev. Lett. 86, 3435 (2001).
[6] A. Meller and D. Branton, Electrophoresis 23, 2583 (2002).

[7] M. Wanunu and A. Meller, Nano Lett. 7, 1580 (2007).

[8] S. M. Iqbal, D. Akin, and R. Bashir, Nat. Nanotechnol. 2, 243 (2007).

[9] A. F. Sauer-Budge, J. A. Nyamwanda, D. K. Lubensky, and D. Branton, Phys. Rev. Lett. 90, 238101 (2003).

[10] J. Mathe, H. Visram, V. Viasnoff, Y. Rabin, and A. Meller, Biophys. J. 87, 3205 (2004).

[11] S. E. Henrickson, M. Misakian, B. Robertson, and J. J. Kasian- 
owicz, Phys. Rev. Lett. 85, 3057 (2000).

[12] J. J. Kasianowicz, S. E. Henrickson, H. H. Weetall, and B. Robertson, Anal. Chem. 73, 2268 (2001).

[13] J. J. Kasianowicz, J. W. F. Robertson, E. R. Chan, J. E. Reiner, and V. M. Standford, Annu. Rev. Anal. Chem. 1, 737 (2008).

[14] V. M. Stanford and J. J. Kasianowicz, Transport of DNA through a Single Nanometer-scale Pore: Evolution of Signal Structure, in Proceedings of the IEEE Workshop on Genomic Signal Processing and Statistics, Baltimore, MD, 2004 (unpublished). See also http://www.itl.nist.gov/iad/IADpapers/2004/ GENSIPS_2004_VMS_JJK.pdf

[15] J. L. Li, D. Stein, C. McMullan, D. Branton, M. J. Aziz, and J. A. Golovchenko, Nature (London) 412, 166 (2001).

[16] J. L. Li, M. Gershow, D. Stein, E. Brandin, and J. A. Golovchenko, Nature Mater. 2, 611 (2003).

[17] D. Fologea, J. Uplinger, B. Thomas, D. S. McNabb, and J. L. Li, Nano Lett. 5, 1734 (2005).

[18] U. F. Keyser, J. B. M. Koelman, S. van Dorp, D. Krapf, R. M. M. Smeets, S. G. Lemay, N. H. Dekker, and C. Dekker, Nat. Phys. 2, 473 (2006).

[19] U. F. Keyser, J. van der Does, C. Dekker, and N. H. Dekker, Rev. Sci. Instrum. 77, 105105 (2006).

[20] C. Dekker, Nat. Nanotechnol. 2, 209 (2007).

[21] E. H. Trepagnier, A. Radenovic, D. Sivak, P. Geissler, and J. Liphardt, Nano Lett. 7, 2824 (2007).

[22] A. J. Storm, J. H. Chen, X. S. Ling, H. W. Zandbergen, and C. Dekker, Nature Mater. 2, 537 (2003).

[23] A. J. Storm, J. H. Chen, H. W. Zandbergen, and C. Dekker, Phys. Rev. E 71, 051903 (2005).

[24] A. J. Storm, C. Storm, J. Chen, H. Zandbergen, J.-F. Joanny, and C. Dekker, Nano Lett. 5, 1193 (2005).

[25] S. M. Simon, C. S. Peskin, and G. F. Oster, Proc. Natl. Acad. Sci. U.S.A. 89, 3770 (1992).

[26] W. Sung and P. J. Park, Phys. Rev. Lett. 77, 783 (1996).

[27] P. J. Park and W. Sung, J. Chem. Phys. 108, 3013 (1998).

[28] E. A. diMarzio and A. L. Mandell, J. Chem. Phys. 107, 5510 (1997).

[29] M. Muthukumar, J. Chem. Phys. 111, 10371 (1999).

[30] M. Muthukumar, J. Chem. Phys. 118, 5174 (2003).

[31] C. Y. Kong and M. Muthukumar, Electrophoresis 23, 2697 (2002); J. Chem. Phys. 120, 3460 (2004); J. Am. Chem. Soc. 127, 18252 (2005); M. Muthukumar and C. Y. Kong, Proc. Natl. Acad. Sci. U.S.A. 103, 5273 (2006); C. Forrey and Muthukumar, J. Chem. Phys. 127, 015102 (2007); C. T. A. Wong and M. Muthukumar, ibid. 128, 154903 (2008).

[32] D. K. Lubensky and D. R. Nelson, Biophys. J. 77, 1824 (1999).

[33] Y. Kafri, D. K. Lubensky, and D. R. Nelson, Biophys. J. 86, 3373 (2004).

[34] E. Slonkina and A. B. Kolomeisky, J. Chem. Phys. 118, 7112 (2003); S. Kotsev and A. B. Kolomeisky, ibid. 125, 084906 (2006); A. Mohan, A. B. Kolomeisky, and M. Pasquali, ibid. 128, 125104 (2008).

[35] S. Matysiak, A. Montesi, M. Pasquali, A. B. Kolomeisky, and C. Clementi, Phys. Rev. Lett. 96, 118103 (2006).

[36] T. Ambjornsson, S. P. Apell, Z. Konkoli, E. A. DiMarzio, and J. J. Kasianowicz, J. Chem. Phys. 117, 4063 (2002).

[37] R. Metzler and J. Klafter, Biophys. J. 85, 2776 (2003).

[38] T. Ambjornsson and R. Metzler, Phys. Biol. 1, 77 (2004).

[39] T. Ambjornsson, M. A. Lomholt, and R. Metzler, J. Phys.:
Condens. Matter 17, S3945 (2005).

[40] A. Baumgartner and J. Skolnick, Phys. Rev. Lett. 74, 2142 (1995).

[41] J. Chuang, Y. Kantor, and M. Kardar, Phys. Rev. E 65, 011802 (2001).

[42] Y. Kantor and M. Kardar, Phys. Rev. E 69, 021806 (2004).

[43] J. K. Wolterink, G. T. Barkema, and D. Panja, Phys. Rev. Lett. 96, 208301 (2006); D. Panja, G. T. Barkema, and R. C. Ball, J. Phys.: Condens. Matter 19, 432202 (2007); D. Panja and G. T. Barkema, Biophys. J. 94, 1630 (2008).

[44] D. Panja, G. T. Barkema, and R. C. Ball, J. Phys.: Condens. Matter 20, 075101 (2008); H. Vocks, D. Panja, G. T. Barkema, and R. C. Ball, ibid. 20, 095224 (2008).

[45] J. L. A. Dubbeldam, A. Milchev, V. G. Rostiashvili, and T. A. Vilgis, Phys. Rev. E 76, 010801(R) (2007).

[46] J. L. A. Dubbeldam, A. Milchev, V. G. Rostiashvili, and T. A. Vilgis, Europhys. Lett. 79, 18002 (2007).

[47] A. Milchev, K. Binder, and A. Bhattacharya, J. Chem. Phys. 121, 6042 (2004).

[48] K. F. Luo, T. Ala-Nissila, and S. C. Ying, J. Chem. Phys. 124, 034714 (2006).

[49] K. F. Luo, I. Huopaniemi, T. Ala-Nissila, and S. C. Ying, J. Chem. Phys. 124, 114704 (2006).

[50] I. Huopaniemi, K. F. Luo, T. Ala-Nissila, and S. C. Ying, J. Chem. Phys. 125, 124901 (2006).

[51] I. Huopaniemi, K. F. Luo, T. Ala-Nissila, and S. C. Ying, Phys. Rev. E 75, 061912 (2007).

[52] K. F. Luo, T. Ala-Nissila, S. C. Ying, and A. Bhattacharya, J. Chem. Phys. 126, 145101 (2007).

[53] K. F. Luo, T. Ala-Nissila, S. C. Ying, and A. Bhattacharya, Phys. Rev. Lett. 99, 148102 (2007).

[54] K. F. Luo, T. Ala-Nissila, S. C. Ying, and A. Bhattacharya, Phys. Rev. Lett. 100, 058101 (2008).

[55] K. F. Luo, T. Ala-Nissila, S. C. Ying, and A. Bhattacharya, Phys. Rev. E 78, 061911 (2008).

[56] K. F. Luo, S. T. T. Ollila, I. Huopaniemi, T. Ala-Nissila, P. Pomorski, M. Karttunen, S. C. Ying, and A. Bhattacharya, Phys. Rev. E 78, 050901(R) (2008); K. F. Luo, T. Ala-Nissila, S. C. Ying, P. Pomorski, and M. Karttunen, e-print arXiv:0709.4615.

[57] K. F. Luo, R. Metzler, T. Ala-Nissila, and S. C. Ying (unpublished).

[58] S. Guillouzic and G. W. Slater, Phys. Lett. A 359, 261 (2006); M. G. Gauthier and G. W. Slater, Eur. Phys. J. E 25, 17 (2008); M. G. Gauthier and G. W. Slater, J. Chem. Phys. 128, 065103 (2008).

[59] S.-S. Chern, A. E. Cardenas, and R. D. Coalson, J. Chem. Phys. 115, 7772 (2001).

[60] H. C. Loebl, R. Randel, S. P. Goodwin, and C. C. Matthai, Phys. Rev. E 67, 041913 (2003).

[61] R. Randel, H. C. Loebl, and C. C. Matthai, Macromol. Theory Simul. 13, 387 (2004).

[62] Y. Lansac, P. K. Maiti, and M. A. Glaser, Polymer 45, 3099 (2004).

[63] Z. Farkas, I. Derenyi, and T. Vicsek, J. Phys.: Condens. Matter 15, S1767 (2003).

[64] P. Tian and G. D. Smith, J. Chem. Phys. 119, 11475 (2003).

[65] Y. D. He, H. J. Qian, Z. Y. Lu, and Z. S. Li, Polymer 48, 3601 (2007); Y. C. Chen, C. Wang, and M. Luo, J. Chem. Phys. 127, 044904 (2007); Y. J. Xie, H. Y. Yang, H. T. Yu, Q. W. Shi, 
X. P. Wang, and J. Chen, ibid. 124, 174906 (2006).

[66] D. Wei, W. Yang, X. Jin, and Q. Liao, J. Chem. Phys. 126, 204901 (2007).

[67] M. B. Luo, Polymer 48, 7679 (2007).

[68] R. Zandi, D. Reguera, J. Rudnick, and W. M. Gelbart, Proc. Natl. Acad. Sci. U.S.A. 100, 8649 (2003).

[69] S. Tsuchiya and A. Matsuyama, Phys. Rev. E 76, 011801 (2007).

[70] A. Bhattacharya et al. (unpublished).

[71] J. W. F. Robertson, C. G. Rodrigues, V. M. Standford, K. A. Rubinson, O. V. Krasilnikov, and J. J. Kasianowicz, Proc. Natl. Acad. Sci. U.S.A. 104, 8207 (2007).

[72] O. V. Krasilnikov, C. G. Rodrigues, and S. M. Bezrukov, Phys.
Rev. Lett. 97, 018301 (2006).

[73] P. G. de Gennes, Scaling Concepts in Polymer Physics (Cornell University Press, Ithaca, NY, 1979).

[74] M. Doi and S. F. Edwards, The Theory of Polymer Dynamics (Clarendon, Oxford, 1986).

[75] M. P. Allen and D. J. Tildesley, Computer Simulation of Liquids (Oxford University Press, Oxford, 1987).

[76] $W$ is measured from the center of the wall particles. For a value of $W=3 \sigma$, the actual separation between the outermost boundaries of the two walls is $2 \sigma$.

[77] D. L. Ermak and H. Buckholz, J. Comput. Phys. 35, 169 (1980).

[78] E. Granato and S. C. Ying, Phys. Rev. Lett. 85, 5368 (2000). 